Knight $^{11}$ has reported the presence of lysine with other amino-acids in the nitrogen content of tobacco mosaic virus. It is possible that the new band having $R_{F}$ value $0 \cdot 20$ might be the lysine of tobacco mosaic virus protein. However, the formation of asparagine and the increase in the content of other free aminoacids, specially aspartic acid, in the diseased leaves supports Bawden's view ${ }^{12}$ that virus infection leads to the formation of a new range of proteins.

We are grateful to Prof. Shri Ranjan for his suggestions and for laboratory facilities. We thank also Sri K. S. Bilgrami for his help in autoclaving the solutions.

Department of Botany,

M. M. Laloraya GOVIND JEE

University of Allahabad.

1 Martin, L. F., Balls, A. K., and McKinney, H. H., Science, 87, 177 (1951).

${ }^{2}$ Meneghini, M., and Delwiche, C. C., J. Biol. Chem., 187, 177 (1951).

${ }^{3}$ Commoner, B., and Nehari, V., J. Gen. Physiol., 36 (6), 791 (1953).

4 Takahashi, W. N., and Mamoru, I., Nature, 169, 419 (1952).

${ }_{5}$ Commoner, B., Newmark, P., and Rodenberg, S. D., Arch. Biochem. and Biophys., 37 (7), 15 (1952).

- Wildman, S. G., Cheo, C. C., and Bonner, J., J. Biol. Chem., 180, 985 (1949).

${ }^{7}$ Giri, K. V., and Rao, N. A. N., Nature, 169, 923 (1954).

${ }^{8}$ Giri, K. V., Krishnamurthy, K., and Vekatasubramanian, T. A., Curr. Sci., 21 (2), 44 (1952).

- Ganguli, N. C., Nature, 174, 189 (1954).

${ }^{10}$ Ranjan, S., Govindjee, and Laloraya, M. M., Proc. Nat. Inst. Sci., India (in the press).

$"$ Knight, C. A., J. Biol. Chem., 171, 297 (1947).

12 Bawden, F. C., J. Sci. and Indust. Res., 13 (3), 106 (1954).

\section{Extreme Resolution of Infra-Red Absorption Spectra}

Rank and Wiggins have constructed an infra-red spectrometer with a plane grating of fine quality and 10-metre optics, double-passed ${ }^{1}$.

An ideal subject with which to study the resolution was found in the $l$-doublets of the $\pi \rightarrow \pi$ band of hydrogen cyanide at $1 \cdot 5 \mu$. The components of these doublets are equal in intensity and their separations are proportional to the $J$ numbers ${ }^{2}$. Moreover, the separations are known, so that a distinction can be made between the conditions 'just resolved' and 'fully resolved'. When a doublet is 'just resolved', the structure is recognized but the apparent separation between the components is smaller than the true value. For example, $P(7)$ was just resolved with a measured separation of $0.072 \mathrm{~cm} .^{-1}$ as compared with the actual value of $0.096 \mathrm{~cm}^{-1}$. The closest doublets to be fully resolved were $P(15)$ and $R(9)$, the true separations of which are $0.180 \mathrm{~cm}^{-1}$ and $0.173 \mathrm{~cm} .^{-1}$ respectively.

While this performance exceeded that of any other existing grating instrument, it was deemed of interest to investigate the possibility of increasing the resolution even further. Since the limit of resolution was set, not by lack of energy or by insufficient sensitivity of the detectors but by imperfections in the grating itself, nothing was to be gained by additional passes through the optical system. Indeed, since it was unlikely that gratings of substantially better quality would be produced in the near future, it was clear that improvement could not be obtained with any grating instrument alone, no matter what its design.

A method for using a Fabry-Perot interferometer in infra-red absorption studies has already been described ${ }^{3}$. The technique had been developed originally to improve the resolution of small spectrometers; but it was superseded by the device of multiple-passing*. It was found to be tedious and impractical by comparison. However, the interferometer is very suitable for our present purpose.

A Fabry-Perot interferometer was crossed with the grating spectrometer. The separation of the interferometer plates was set so that the range of frequency between orders was equal to the spectral slitwidth of the spectrometer, which thus served as a primary monochromator the function of which was to remove overlapping orders. If a fraction $\Delta m$ of a fringe order could be resolved, then under the above conditions the resolution of the combination of the interferometer and spectrometer was as much as $1 / \Delta m$ times that of the latter alone. In this way it was possible to resolve fully the doublets $P(3)$ and $R(2)$; the measured separation of $R(2)$ was $0.045 \mathrm{~cm}^{-1}$. This is seen to represent a sixfold improvement if the Doppler width $\left(0.016 \mathrm{~cm}^{-1}\right)$ of the lines is taken into account.

The effective reflectivity of the plates was 65 per cent, produced by zinc sulphide - magnesium fluoride coatings. The plate separation was $28.5 \mathrm{~mm}$.

The purpose of this communication is to direct attention to the fact that it is now possible to attain a full resolving power of well in excess of 200,000 at $1 \cdot 5 \mu$. This may be near the useful limit at the present time, because of Doppler broadening of the absorption lines. Nevertheless, the system has still not reached its energy limit, and the resolution could be improved further by increasing the separation between the plates of the interferometer. Full details of the instrument will be published elsewhere.

This research was assisted by support from Contract N6onr Task V of the U.S. Office of Naval Research.
J. H. JAFFE*
T. A. Wiggins
D. H. RANK

Physics Department,

Pennsylvania State University,

State College, Pennsylvania.

$$
\text { Jan. } 5 .
$$

* On sabbatical leave from the Weizmann Institute of Science, Rehovoth, Israel.

${ }^{1}$ Rank, D. H., and Wiggins, T. A., J. Opt. Soc. Amer., 42, 983 (1952). ${ }^{2}$ Wankgins, Shearer, Shull and Rank, J. Chem. Phys., 22, 547 (1954).

${ }^{3}$ Jaffe, J. H., Nature, 168, 381 (1951).

4 Walsh, A., Nature, 16\%, 810 (1951).

\section{A Solar Noise Outburst of January 15, 1955}

AN interferometer-type noise receiver has been operating at the Radio Physics Laboratory of the Research Defence Board, Canada, for more than a year. This receiver has been recording the radiation at a frequency of $50 \mathrm{Mc} . / \mathrm{s}$. from the discrete source in the constellation of Cassiopeia. However, the antenna is so constructed that solar noise will be recorded if of sufficient magnitude.

On January 15 of this year a particularly large noise outburst from the sun was observed. The outburst commenced at 9.45 a.m. Eastern Standard Time as a single spike on our record, which lasted approximately four minutes. This was followed at $9.53 \mathrm{a} . \mathrm{m}$. by a large increase in the noise-level lasting until 3.00 p.m. On the following day a very large burst was again observed, commencing at 